\title{
Editorial: Novel Tools for the Study of Structural and Functional Networks in the Brain
}

\author{
Luis M. Colon-Perez ${ }^{1 *}$, Thomas Mareci ${ }^{2}$ and Mingzhou Ding ${ }^{3}$ \\ ${ }^{1}$ Department of Psychiatry, University of Florida, Gainesville, FL, United States, ${ }^{2}$ Department of Biochemistry and Molecular \\ Biology, University of Florida, Gainesville, FL, United States, ${ }^{3}$ Department of Biomedical Engineering, University of Florida, \\ Gainesville, FL, United States
}

Keywords: neuroimaging, fMRI methods, tractography, connectivity, neuroscience methods

\section{Editorial on the Research Topic}

\section{Novel Tools for the Study of Structural and Functional Networks in the Brain}

Throughout the history of neuroscience, technological advances are the drivers behind many major advances in our understanding of the nervous system. Early optical investigations led to the development of the microscope, and quantum mechanics led to nuclear magnetic resonance, which is the foundation of magnetic resonance imaging (MRI). Recent progress in MRI technology has allowed the elucidation of the complex organization and function of brain networks with unprecedented spatial and temporal precision. It is not an exaggeration to say that characterizing the intricate architecture and dynamics of the brain is one of the leading frontiers in modern science. Neuroscience offers an exceptional opportunity for interdisciplinary research where biology, physics, mathematics, and engineering come together to advance boundary of the frontier.

Investigations of the structure and function of the brain take place on multiple scales, including macroscale at the level of brain regions, mesoscale at the level of neuronal populations, and microscale at the level of single neurons and neuron-neuron interactions. Integration of knowledge over these scales requires novel techniques and interpretations. In this research topic, we highlight nine articles that integrate structural and functional approaches to study brain networks.

The research articles contained in this research topic can be divided along three separate sub-topics. The first subtopic features novel parcellations to study connectomes (Figley et al.; Figley et al.; Schiffler et al.). The second sub-topic introduces new methods to analyze functional data (Cheng et al.; Peng et al.; Wu et al.). The third sub-topic applies in vivo methods to analyze brain changes co-occurring with diseases (Huang et al.; James et al.; Liao et al.). The parcellation subtopic starts with a study that combines fMRI and tractography to create probabilistic white matter atlases for each of the six commonly studied resting-state brain networks (Figley et al.). This work introduces a comprehensive set of white matter maps for well-known resting state networks such as dorsal default mode network, ventral default mode network, left executive control network, right executive control network, anterior salience network, and posterior salience network (Figley et al.). The second parcellation article combines fMRI with tractography to create probabilistic white matter atlases for eight functional brain networks (Figley et al.), including auditory, basal ganglia, language, precuneus, sensorimotor, primary visual, higher visual and visuospatial networks (Figley et al.). These white matter atlases in stereotaxic coordinates could be used to associate white matter changes to changes in particular functional brain networks selectively, or compliment resting state fMRI by defining the underlying anatomical pathway that gives rise to functional connectivity. The third parcellation study combines cortical parcellation and tractograms to create a subject-specific white matter parcellation (Schiffler et al.). The parcellation scheme associating large white matter 
areas to specific cortical regions allows us to relate white matter alterations to alterations to specific cortical regions (Schiffler et al.).

The functional sub-topic starts with an article describing a pseudo-bootstrap (PBS) analysis that can be used to avoid using template-based parcellations schemes in studies of brain network analysis (Cheng et al.). This pseudo-bootstrap method can identify individuals across scan sessions based on the mean functional connectivity with an accuracy rate of $\sim 90 \%$ (Cheng et al.). It does so by finding the maximum correlation of mean functional connectivity of pseudo-bootstrap samples between two scan sessions. The second functional article describes a novel measure for functional segregation of the brain by employing a frequency clustering analysis method based on Hilbert-Huang Transform (HHT) in conjunction with a label-replacement procedure (Wu et al.). This HHT scheme provides a novel measure for functional segregation of the brain according to time-frequency characteristics of resting state BOLD activities, and is robust, yielding almost identical clusters when applied to different runs of a dataset or different datasets (Wu et al.). The third functional article develops a rodent perfusion autoradiograph toolbox to study connectivity in mesoscale data (Peng et al.). This toolbox allows for sampling of standardized data from images of brain slices, as well as provides a way to analyze and display functional connectivity data in the rat cerebral cortex (Peng et al.).

The application sub-topic starts with a study using positron emission tomography (PET) to quantify selective serotonin reuptake inhibitors (SSRI) to detect changes in interregional correlations of the serotonin transporter binding potential. The results of this study suggest that SSRIs induce interregional changes (i.e., connectivity), rather than mere focal modifications (James et al.).The second application study assessed abnormal spontaneous brain activity and described the intricate neural mechanism of premenstrual syndrome the results. The results suggest that abnormal spontaneous brain activity is found in PMS patients and the severity of symptom is related explicitly to the left MFC and right ACC (Liao et al.). The last article of the application sub-topic aimed to determine whether patients with Primary blepharospasm exhibit altered functional brain connectivity. This study found many differences in multiple neural networks in primary BPS (Huang et al.).

In closing, this research topic includes nine excellent articles on a wide array of methods and applications in both human and animal neuroscience. The editors thank the authors for their multifaceted contributions to advance our understanding of the brain.

\section{AUTHOR CONTRIBUTIONS}

All authors listed have made a substantial, direct and intellectual contribution to the work, and approved it for publication.

Conflict of Interest Statement: The authors declare that the research was conducted in the absence of any commercial or financial relationships that could be construed as a potential conflict of interest.

Copyright (c) 2018 Colon-Perez, Mareci and Ding. This is an open-access article distributed under the terms of the Creative Commons Attribution License (CC $B Y)$. The use, distribution or reproduction in other forums is permitted, provided the original author(s) and the copyright owner are credited and that the original publication in this journal is cited, in accordance with accepted academic practice. No use, distribution or reproduction is permitted which does not comply with these terms. 